\title{
Design of a Smart Home Remote Monitoring System Based on $\mathrm{C}++$ and ZigBee
}

\author{
Xuewen Shi ${ }^{1}$, Qinglan Wang ${ }^{1, *}$, Guangdeng Zong ${ }^{1}$ and Tongdui Zhang ${ }^{2}$ \\ ${ }^{1}$ Engineering College, Qufu Normal University, Shandong 276826, China \\ ${ }^{2}$ Institute of Scientific and Technical Information of Rizhao, Rizhao Shandong, 276826, China \\ ${ }^{*}$ Corresponding author
}

\begin{abstract}
In this paper, a smart home remote monitoring system was designed based on $\mathrm{C}++$ and ZigBee. The system is composed of slave subsystem and host subsystem. The slave subsystem is a wireless sensor network designed by using ZigBee, it performs the real-time detection of temperature and humidity, smog, gas concentration and the control of household appliances. The host subsystem consists of two parts, PC client monitoring platform designed by using $\mathrm{C}++$ language and mobile client monitoring platform designed based on Android. Communication via WLAN, the two platforms achieved remote monitoring of home together. Finally, testing results show that the system has advantages of good stability, low cost, powerful and convenient operation, so it has a better application prospect.
\end{abstract}

Keywords- wireless sensor network; C++; mobile phone APP

\section{INTRODUCTION}

With the rapid development of science and economy, people's pursuit of comfortable life becomes higher and higher, so smart home monitoring system comes into being [1]. However, the cost of smart home is high at present, it is difficult for ordinary families to accept. In this paper, a smart home monitoring system was designed and implemented based on $\mathrm{C}++$ and ZigBee. The slave computer system performs data acquisition and the control of household appliances while the host computer monitoring system performs the real-time display of sense data and the issue of control commands. The system is cost-effective, powerful and convenient operation, it is easy to be accepted by ordinary families.

\section{OVERALl Design OF System}

The system is mainly composed of two parts, slave computer data acquisition subsystem and host computer monitoring subsystem [2].The slave computer system is a wireless sensor network designed by using ZigBee, it performs the real-time detection of temperature and humidity, smog, gas concentration and the control of lamp. The host computer monitoring system consists of two parts, PC client monitoring platform designed by using $\mathrm{C}++$ language and mobile client monitoring platform designed based on Android, the overall design of system is shown in Figure I.

The slave computer system uploads the sensor data to PC monitoring platform, which receives the data and draws them into graph, displays them on screens, so it is very convenient to view. When the measured value exceeds its upper limit, alarm is sent out. Another important function, the PC client can also send commands to remote control household appliances, such as desk lamps, air conditioning, electric curtains and others. The mobile client communicates with PC client through WiFi, and has the same functions as the former.

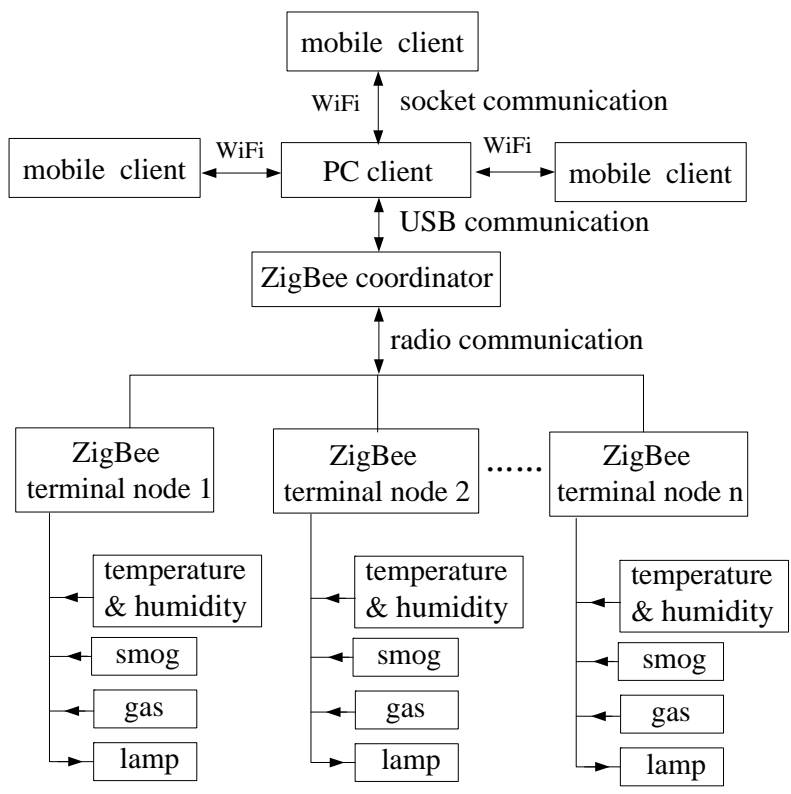

FIGURE I. OVERALL FRAMEWORK OF SYSTEM

\section{HARDWARE CIRCUIT DESIGN OF SYSTEM}

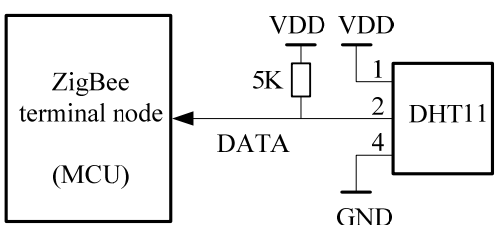

FIGURE II. CIRCUIT SCHEMATIC DIAGRAM OF DTH11

Hardware circuit design of system mainly includes wireless sensor network circuit, sensors interface circuit and household appliance control module circuit, etc. [3] Star topology network is adopted in the wireless sensor network circuit design [4], it includes one coordinator and four terminal nodes which are independently designed by using CC2530 chip and other 
periphery circuits.

DTH11 is a kind of widely used digital temperature and humidity sensor, it directly outputs the measured value in the digital form, and its circuit design schematic diagram is shown in Figure II.

Gas sensor named MQ-2 is used to get values of natural gas and smog concentration, it has advantages of wide detection range, fast response, high stability, what's more, it outputs analog voltage, and can be directly connected to the ADC pin of MCU, so it is easy for us to get the digital value of gas concentration. The circuit interface design is shown in Figure III.

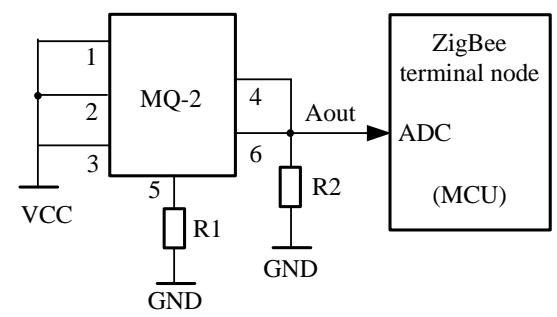

FIGURE III. INTERFACE DESIGN OF MQ-2

The control of household appliances, such as desk lamp, air conditioning, etc, is a switch control. The relay module is often used in process of switch control, its on-off state is controlled by an I/O pin of the CC2530, and the load is controlled by relay. Schematic diagram of the relay control is shown in Figure IV.

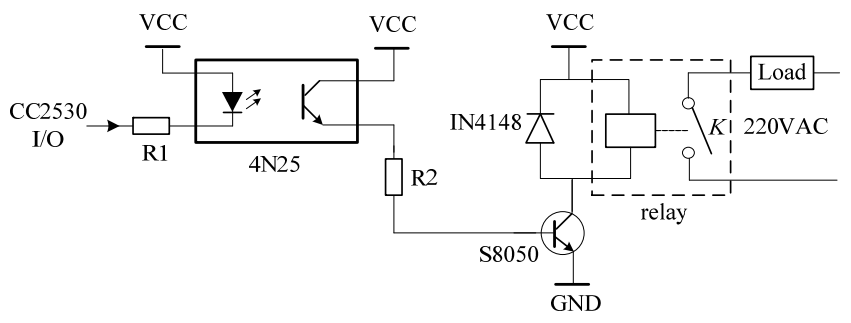

FIGURE IV. CIRCUIT SCHEMATIC DIGRAM OF REALY CONTROL

\section{SOFTWARE DESIGN OF SYSTEM}

Software design includes programming of terminal node, coordinator, PC client monitoring platform and mobile client monitoring platform four parts.

\section{A. Terminal Node Programming}

Terminal node performs data acquisition and the control of household appliances [5]. In order to reduce power consumption and improve stability of system, the method of timing acquisition data and timing transmission data is used on the terminal node, that is, setting a breakpoint in the program of the terminal node, when a task of data acquisition or transmission is done, terminal node begins to be in a state of sleep until the next acquisition time or the control command arrives, then it returns to work. The flow chart of the terminal node programming is shown in Figure $\mathrm{V}$.

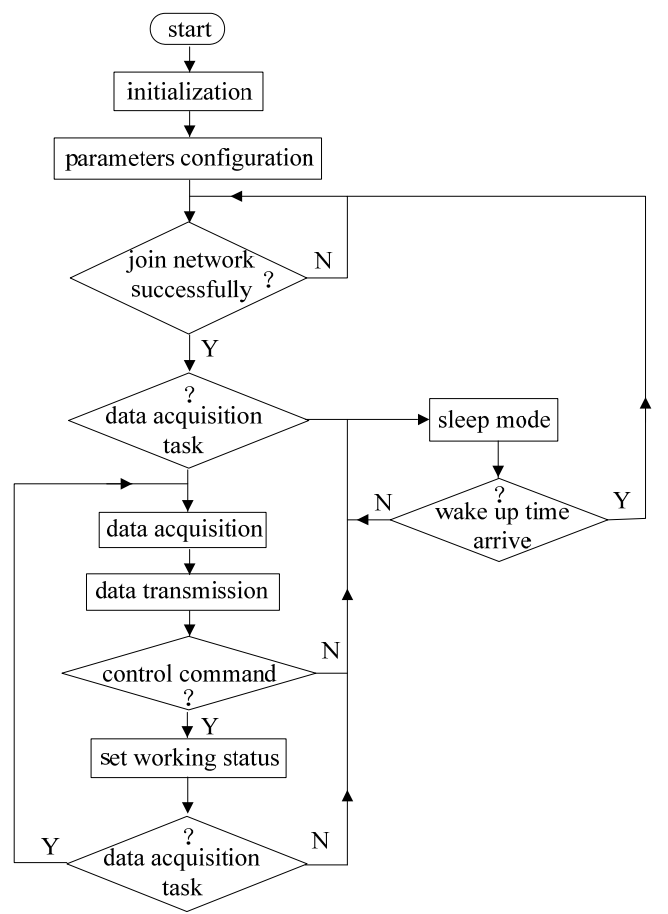

FIGURE V. FLOW CHART OF TERMINAL NODE PROGRAMMING

\section{B. Coordinator Programming}

Coordinator is a key unit of the wireless sensor network [6], which has functions of establishment the wireless network, distribution of the address and data transmission. First, when coordinator is powered on, it performs hardware initialization operation, it can automatically configure the corresponding channel and PAN ID [7].Second, it initializes the operating system, creates wireless sensor network, and waits for terminal nodes to join the network. Last, when network is established, coordinator unit receives data from terminal nodes, and begins to do with it. If the data is OTA (over-the-air technology), coordinator receives it and sends it out through the serial port, else, the data is the control command, it will be sent to terminal nodes in the form of broadcast. The flow chart of the coordinator programming is shown in Figure VI.

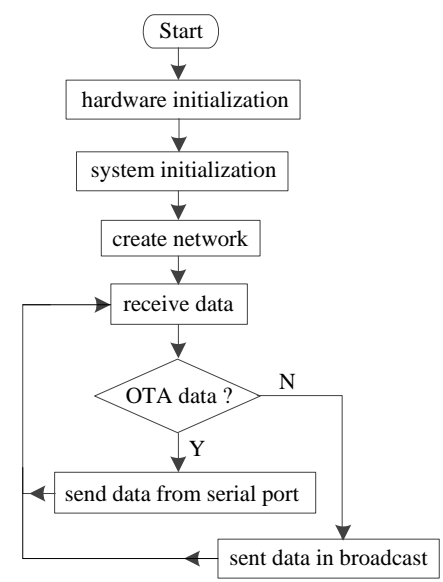

FIGURE VI. FLOW CHART OF COORDINATOR PROGRAMMING 


\section{PC Client Programming}

The PC client monitoring platform is designed with $\mathrm{VC}++6.0$ language, it can receive data from the coordinator through its USB port and display them on screen [8], in addition, it can communicate with the mobile client through Socket function. Flow chart of Socket communication $[9,10]$ is shown in Figure VII.

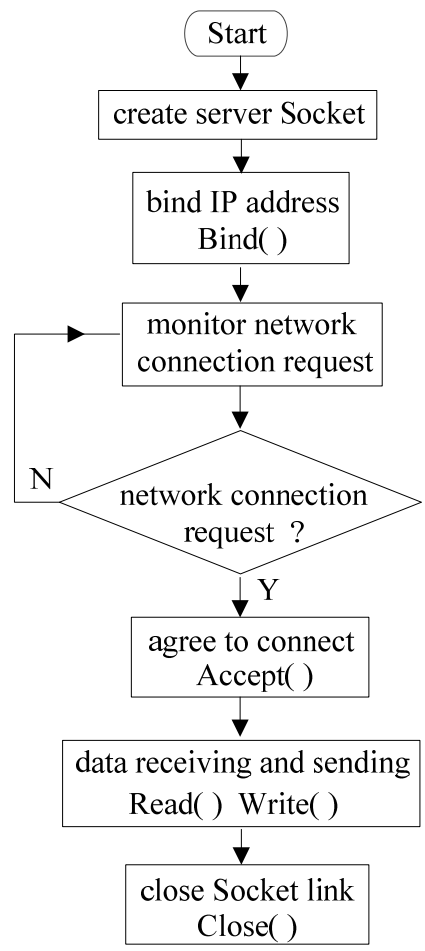

FIGURE VII. FLOW CHART OF PC SOCKET COMMUNICATION

PC client starts to monitor the window after its serial port monitoring program is running, the main program segment is shown as following.

if(m_SerialPort.InitPort(this， nPort，38400， 'N'，8，1， EV_RXCHAR|EV_RXFLAG, 512))

$\{$ m_bComState $=$ TRUE;

m_SerialPort.StartMonitoring( );

m_EditText.SetWindowText("Open the serial port, Baud rate, $384008 \mathrm{~N} 1 ")$;

m_StaticCom.SetBitmap(HBITMAP(m_bmp[0]));

m_btnCom.SetWindowText("Close the serial port "); \}

Host client communicates with mobile client through socket, the main program segment is given below.

$\{$ ServerSock=socket(AF_INET,

SOCK_STREAM, IPPROTO_TCP);

sockaddr_in localaddr;

if(bind(ServerSock,(structsockaddr*)\&localaddr,sizeof(s ockaddr) $)==$ SOCKET_ERROR)

if(WSAAsyncSelect(ServerSock, m_hWnd,
NETWORK_EVENT,FD_ACCEPT | FD_CLOSE |

FD_READ | FD_WRITE) == SOCKET_ERROR)

listen (ServerSock, 5);

return TRUE;

\}

\section{Mobile Client Programming}

Mobile client programming is based on Android4.02 system, including page layout, network connection, Socket communication etc. Page layout is mainly used in combination of table layout and linear layout. Flow chart of the mobile client Socket communication [11] is shown in Figure VIII.

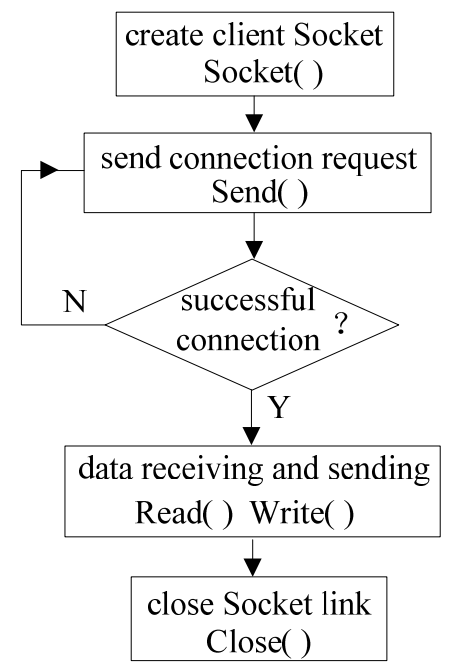

FIGURE VIII. FLOW CHART OF MOBILE PHONE CLIENT SOCKET COMMUNICATION

Open the mobile client APP program, click on the "network setting" button, then input server IP address, its default port is 33333, and then click on the "connection" button to link server. If the link is successful, mobile client may accept data from server or send commands to control household appliances.

\section{SYSTEM TEST}

After the smart home remote monitoring system is established, program is downloaded to terminal nodes and coordinator, and then system starts to be debugged. Physical model of a terminal node is given in Figure IX, the user interface of mobile client is shown in Figure X.

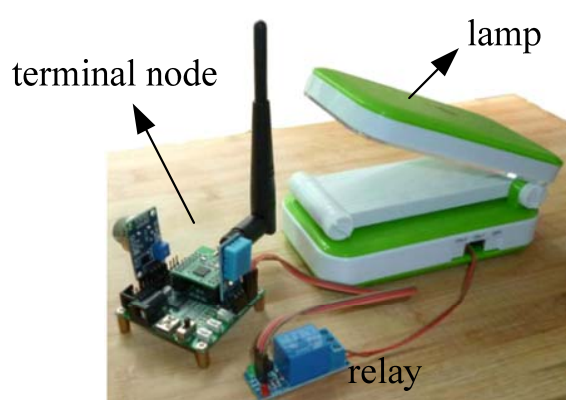

FIGURE IX. PHYSICAL MODEL OF A TERMINAL NODE 

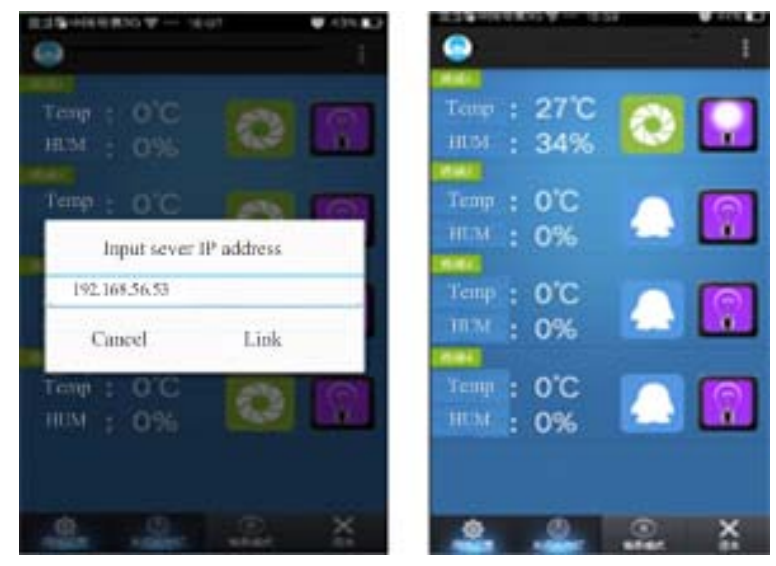

FIGURE X. USER INTERFACE OF MOBILE CLIENT

\section{CONCLUSION}

A smart home remote monitoring system was designed in this paper, it achieved real-time detection and control of home. This system has advantages of good stability, low cost, powerful and convenient operation, etc, so it is easy to be accepted by ordinary families. If the mobile client can achieve remote monitoring in WWW network, it will have a greater value of popularization and application in the future.

\section{ACKNOWLEDGMENT}

The research work was supported by National Natural Science Foundation of China (Grant No.61304059). The authors would like to thank all colleagues who previously provided technical support.

\section{REFERENCES}

[1] X.L. Xu, M. Liu. Design of Domestic Environment Monitoring System Based on ZigBee[J], Electronic Design Engineering. Vol.24, No.1, 152155, 2016.

[2] X.D. Wang. Research and Design of Intelligent Home System Based on STM32 and Zigbee[D], QuFu: QuFu Normal University, 2015.

[3] L. Zhou, H.W. Zhang. Design of Remote Query and Control System Used in Smart Home Based on ZigBee, Transactions on Information and Communication Technologies, Vol. 56, (c) 2014 WIT Press,341$347,2014$.

[4] J.W. Wang. Software Design of Smart Home System Based on Internet of Things[D],JiNan: ShanDong University,2013.

[5] Y. Wu, T. Wu, P. Liu. Smart Home System Based on ZigBee and ARM, The11thIEEE International Conference on Electronic Measurement \& Instruments.772-777,2013.

[6] Z.Y. Liu. Hardware Design of Smart Home System Based on zigBee Wireless Sensor Network, 2014 AASRI Conference on Sports Engineering and Computer Science (SECS 2014),75-81,2014.

[7] X.N. Peng, X.P. Zou, Z.X. Yu, Y. Yang.Design of Household Appliance Control Sysytem Based on ZigBee, Proceedings of IEEE CCIS2012,1486-1490,2012.

[8] Z.J. Wang.VC++6.0 Programming from Entry to Master, Tsinghua university press, Beijing, China,2010.

[9] A.H. Tian,C.B. Fu,L. Li. Research on Network Communication Process Based on Socket[J], Jouranl of QuJiang Normal University, Vol.32, No.6,45-47,2013.

[10] J.L. Wang, Y.H. Wang, L. Tian. Study on Socket Communication Based on WSNs[J], Transducer and Microsystem Technologies, Vol.33,No.2,39-42,2014.
[11] Y. Cui, W.H. Zhang, Y.F. Bai. Intellectual Home Control System Based on Arduino[J], Application of Electronic,Vol.40, No.4,39-42,2014. 